Pacific Journal of Mathematics

SECOND ORDER COMPLEX DIFFERENTIAL EQUATIONS 


\title{
SECOND ORDER COMPLEX DIFFERENTIAL EQUATIONS WITH A REAL INDEPENDENT VARIABLE
}

\author{
JOHN H. BARRETT
}

Introduction. This paper is concerned with the oscillation and boundedness properties of solutions of the complex differential equation

$$
(p(x) \dot{y})^{\bullet}+f(x) y=0,
$$

$a \leqq x<\infty$,

where $p(x)=p_{1}(x)+i p_{2}(x) \neq 0, f(x)=f_{1}(x)+i f_{2}(x)$ and each of the functions $p_{1}(x), p_{2}(x), f_{1}(x)$ and $f_{2}(x)$ is a continuous real function on the half-line $a \leqq x<\infty$.

Such differential equations have many interpretations and applications. For example, if $p(x)=1$ and the real and imaginary parts of equation (1) are separated the resulting system of two real equations can be interpreted as equations of motion in the $y_{1} y_{2}$-plane, where $y=$ $y_{1}+i y_{2}$, as in $[4,9]$. If in (1) $x$ is replaced by the complex variable $z$, and the coefficients are required to be analytic functions of $z$ the resulting completely complex equation can be reduced to one of the type (1) by considering certain analytic paths in the z-plane. This procedure has been used effectively by Taam [9] and others to find zero-free regions for the completely complex equation. Also, Hille [5] has made an extensive study of the behavior of solutions of a special case of (1), where $p(x)=1$ and $f(x)=\lambda F(x), F(x)$ real and positive and $\lambda$ a complex parameter, and has used these results in his study of Cauchy's problem for a generalized heat equation.

The present study of equation (1) begins with consideration of the special case

$$
\left(\dot{y}\{q(x))^{\cdot}+\bar{q}(x) y=0\right.
$$$$
\alpha \leqq x<\infty,
$$

where $q(x)=q_{1}(x)+i q_{2}(x) \neq 0, q_{1}(x)$ and $q_{2}(x)$ are real and continuous on $a \leqq x<\infty$ and $\bar{q}$ is the complex conjugate of $q$. For $q(x)$ real a fundamental set of solutions consists of $\sin \int_{a}^{x} q$ and $\cos \int_{a}^{x} q$. This suggests an investigation of the corresponding complex solutions, $s[a, x ; q]$ and $c[a, x ; q]$, of (2) when $q$ is complex. These "trigonometric" functionals satisfy identities and inequalities analogous to those of the real sines

Received June 5, 1957. This work was partially supported by the National Science Foundation (NSF-G-1825) through the University of Delaware and was begun while the author was a lecturer at Yale University.

Presented to the American Mathematical Society December 1956. 
and cosines. For example, the sum of the squares of the magnitudes is identically one and, hence, all solutions of equation (2) are bounded on $a \leqq x<\infty$. This boundedness property is the main point of departure from the analytic definition of trigonometric functions of a complex variable $z$, where $\sin \int_{a}^{x} q$ is unbounded if $\int_{a}^{x} q_{2}$ is unbounded. The boundedness property is useful in the applications of the last section.

An additional advantage in considering the special case is that for a rather large class of coefficient functions, $q(x)$, equation (2) can be solved explicitly, thus providing a new set of much needed examples to give insight into the oscillatory behavior of solutions of (1). Furthermore, for a still larger class of coefficient functions the oscillatory behavior of solutions of (2) is determined. An interesting result is that the zero separation properties, true for the real case, are often violated for the complex equation (2). For example, a class of functions, $q(x)$, is found for which the "sine" $s[a, x ; q]$ oscillates (has infinitely many zeros on $a \leqq x<\infty)$ and the " cosine" $c[a, x ; q]$ has no zero on $a \leqq x<\infty$.

The final section shows that although equation (2) is a special case of (1), all oscillatory behavior patterns of equations of the type (1) are present in those of type (2). In particular, for each non-trivial solution $y(x)$ of (1), for which $y(a)=0$, and each non-zero function $w(x)$ of class $C^{\prime}$ there exist a continuous complex coefficient function $q(x)$ and a nonzero "amplitude" function $\rho(x)$ of class $C^{\prime}$ such that

$$
y(x)=\rho(x) s[a, x ; q], \quad p(x) \dot{y}(x)=\bar{w}(x) \bar{\rho}(x) \bar{c}[a, x ; q] .
$$

For $w(x), p(x)$ and $f(x)$ real ; $q(x)$ and $\rho(x)$ are real and (3) reduces to the modified Prüfer transformation $[1,6]$

$$
y=\rho(x) \sin \theta(x), p \dot{y}=w(x) \rho(x) \cos \theta(x), \theta(x)=\int_{a}^{x} q,
$$

which has been useful in establishing real oscillation and boundedness theorems. The author [3] has developed a Prüfer transformation for equations of the form of (2) with both coefficients being square symmetric matrices and a similar, but less useful, transformation of type (3) (that is $y=\rho s, p \dot{y}=\rho c$ ) can be obtained as a corollary of the matrix theorems.

Since the "amplitude" $\rho(x)$ is non-zero and the "sine" $s[a, x ; q]$ is bounded, the Prüfer-type transformation (3) separates boundedness considerations from those of oscillation, as does (4) for the real case. Applications of (3) yield bounds on solutions of (1) of the LiapounoffBirkhoff-Levinson type. For the special case, $p(x)=1$ and $w(x)$ a positive real constant, these exponential bounds reduce to those of Taam [7]. It it noted that Taam failed to achieve a "symmetric" form because he 
specialized $p(x)$ to be real and, in particular, $p(x)=1$.

Further study of the relation of $q(x)$ to the original coefficients, $p(x)$ and $f(x)$, and of the oscillatory properties of the functionals of the first section should lead to new oscillation theorems for (1).

1. Complex trigonometry. Let $q(x)=q_{1}(x)+i q_{2}(x), q_{1}$ and $q_{2}$ be continuous functions on $a \leqq x<\infty$ and define $c=c(x)=c[a, x ; q], s=s(x)=$ $s[a, x ; q]$ to be a solution (pair) of the complex first order system

$$
\begin{array}{ll}
\dot{s}=q \bar{c}, & s(a)=0, \\
\dot{c}=-q \bar{s}, & c(a)=1 .
\end{array}
$$

If, in addition, $q(x) \neq 0$ it is easily seen that $s$ and $c$ are solutions of the second order equation

$$
(\dot{y} / q)^{\cdot}+\bar{q} y=0
$$

with initial conditions

$$
\begin{aligned}
& s(a)=0, c(a)=1, \\
& \dot{s}(a)=q(a), \dot{c}(a)=0 .
\end{aligned}
$$

Note that $s=\sin \int_{a}^{x} q$ and $c=\cos \int_{a}^{x} q$, if $q(x)$ is real and, furthermore, if $q \neq 0$ both solutions oscillate (have infinitely many zeros on $a \leqq x<\infty$ ) only if $\int_{a}^{\infty}|q|=\infty$.

Boundedness is retained for complex $q(x)$, as is seen by :

LeMma 1.1. $|s|^{2}+|c|^{2}=1$.

Proof. Differentiate $s \bar{s}+c \bar{c}$ and note its value at $x=a$.

There is also an extension of the properties that the real sine function is odd and the cosine is even. This result is useful in carrying out the details of the proof of Theorem 1.2.

Lemma 1.2. If $k$ is a complex number such that $|k|=1$ then $s[a, x ; k q]=k s[a, x ; q]$ and $c[a, x ; k q]=c[a, x ; q]$ on $a \leqq x<\infty$.

Proof. Let $m(x)=s[a, x ; k q]-k s[a, x ; q]$ and $n(x)=c[a, x ; k q]-$ $c[a, x ; q]$.

Then $m(a)=0, n(a)=0$ and $\dot{m}=k q \bar{n}, \dot{n}=-k q \bar{m}$, whose only solution is $m \equiv n \equiv 0$ on $a \leqq x<\infty$, thus completing the proof.

Consider now the polar form of solutions of (5) and (2) in terms of the polar components of the coefficients; i. e., suppose

$$
q(x)=r(x) \exp (i \theta(x)), \quad a \leqq x<\infty,
$$


where $r(x)$ is real, continuous and positive and $\theta(x)$ is real and of class $C^{\prime}$. These conditions ensure a polar form for the complex trigonometric functionals as is seen by the following.

LEMMA 1.3. Under the above hypotheses on $q(x)$, there exist on $a \leqq x<\infty$ real functions $h(x)$ and $\alpha(x)$ such that $h, \dot{h} / r, \alpha$ and $h \dot{\alpha} / r$ are of class $C^{\prime}$ and, furthermore

$$
s[a, x ; q]=h(x) \exp (i \alpha(x)) .
$$

Proof. Let $s(x)=s[a, x ; q]$. Using a technique similar to that employed by Taam [9], define the real function

$$
g(x)=\left\{\begin{array}{lll}
\Im(\dot{s} / r s), & \text { if } & s(x) \neq 0 \\
\dot{\theta} / 2 r, & \text { if } & s(x)=0
\end{array}\right.
$$

Note that $g(x)$ is continuous on $a \leqq x<\infty$, since computation by means of L'Hopitals rule shows

$$
\lim _{x \rightarrow x_{0}} \Im(\dot{s} / r s)=\dot{\theta}\left(x_{0}\right) / 2 r\left(x_{0}\right) \text {, if } s\left(x_{0}\right)=0 .
$$

Let

$$
\alpha(x)=\theta(a)+\int_{a}^{x} r(t) g(t) d t,
$$

then $\alpha(x)$ is of class $C^{\prime}$ on $a \leqq x<\infty$. Let

$$
h(x)=s(x) \exp (-i \alpha(x)),
$$

then $h(x)$ is of class $C^{\prime}, h(a)=0$ and

$$
\dot{h}(x)=(\dot{s}-i \dot{\alpha} s) \exp (-i \alpha) \text {. }
$$

Thus, $\dot{h}(a)=\dot{s}(a) \exp (-i \alpha(a))=r(a)>0$.

The next step is to prove that $h=h_{1}(x)+i h_{2}(x)$ is real, that is, $h_{2}(x) \equiv 0$. Suppose $h_{2}(x) \not \equiv 0$, then there exist numbers $t_{0}<t_{1}$ such that $h_{2}\left(t_{0}\right)=0$, $h_{2}(x) \neq 0$ on $t_{0}<x<t_{1}$ and $s(x) \neq 0$ on $t_{0}<x<t_{1}$. Then on $t_{0}<x<t_{1}$

$$
\frac{\dot{h}}{h}=\frac{\exp (i \alpha) \dot{h}}{s}=\frac{\dot{s}}{s}-i \dot{\alpha},
$$

Also,

$$
\Im\left(\frac{\dot{h}}{h}\right)=\frac{\Im(\bar{h} \dot{h})}{|s|^{2}}=0
$$




$$
h_{1} \dot{h}_{2}-h_{2} \dot{h}_{1}=0 \text {. }
$$

Therefore there exists a real constant $k$ such that $h_{1}(x)=k h_{2}(x)$ on $t_{0}<$ $x<t_{1}$. Herce $h_{1}\left(t_{0}\right)=h_{1}\left(t_{0}+\right)=k h_{2}\left(t_{0}+\right)=0$ and $s\left(t_{0}\right)=0$. Suppose $t_{0}=a$, then, since $h(a)$ is real, $\dot{h}_{2}\left(t_{0}\right)=0$ and $\dot{h}_{1}\left(t_{0}\right)=\dot{h}_{1}\left(t_{0}+\right)=k \dot{h}_{2}\left(t_{0}\right)$. But this requires that $\dot{s}\left(t_{0}\right)=0$, which contradicts the fact that $s(x)$ is non-trivial. In a similar manner and by use of an induction argument it is easily seen that $t_{0}$ cannot be any zero of $s(x)$. Thus, $h_{2}(x) \equiv 0$ and $h(x)$ as defined by (11) is real.

Recall that $\dot{s}=q \bar{c}$ and $\dot{c}=-q \bar{s}$ where $s=s[a, x ; q]$ and $c=c[a, x ; q]$ and $s=h(x) \exp (i \alpha(x))$, where $q=r(x) \exp (i \theta(x))$. By differentiating this polar form of $s$ and simplifying it follows that

$$
\frac{\dot{h}}{r}+i \frac{\dot{h \alpha}}{r}=\bar{c} \exp (i(\theta-\alpha))
$$

and, since the right hand side is of class $C^{\prime}$, that the real components, $\dot{h} / r$ and $h \dot{\alpha} / r$ are likewise of class $C^{\prime}$. Furthermore, by transposing the exponential factor to the left hand side and differentiating we obtain

$$
\left(\frac{\dot{h}}{r}\right)^{\cdot}+i\left(\frac{\dot{h} \dot{\alpha}}{r}\right)^{\cdot}+i(\dot{\alpha}-\dot{\theta}) \frac{\dot{h}}{r}-(\dot{\alpha}-\dot{\theta}) \frac{h \dot{\alpha}}{r}=-r h .
$$

Separation into real and imaginary parts yields the system

$$
\left(\frac{\dot{h}}{r}\right)^{\cdot}+\left(1-\frac{\dot{\alpha}(\dot{\alpha}-\dot{\theta})}{r^{2}}\right) r h=0,
$$

and

$$
\left(\frac{h \dot{\alpha}}{r}\right)^{\cdot}+\frac{\dot{\alpha}-\dot{\theta}}{r} \dot{h}=0
$$

thus completing the proof of Lemma 1.3.

Integration of equation (13) gives

$$
\frac{h^{2}(x) \dot{\alpha}(x)}{r(x)}=\int_{a}^{x} \frac{h \dot{h} \dot{\theta}}{r} .
$$

Finally, integration by parts establishes the following.

Lemma 1.4. If, in addition to the hypotheses of Lemma 1.3, the quotient $\dot{\theta} / r$ is of class $C^{\prime}$, then on $a \leqq x<\infty$ 


$$
\frac{h^{2}(x)}{r(x)}\left(\dot{\alpha}(x)-\frac{\dot{\theta}(x)}{2}\right)=-\frac{1}{2} \int_{a}^{x} h^{2}(t)\left(\frac{\dot{\theta}(t)}{r(t)}\right) \dot{d} d t
$$

Furthermore, $\dot{\alpha} / r$ is of class $C^{\prime}$, whenever $s \neq 0$.

The preceding discussion suggests special consideration of the quotient $\dot{\theta} / r$. The following theorem is a compilation of the results thus far.

THeOREM 1.1. If on $a \leqq x<\infty, q=r(x) \exp (i \theta(x)), r(x)$ is real, positive and continuous, $\theta(x)$ is real and of class $C^{\prime}, b(x)=\dot{\theta}(x) / 2 r(x)$, then there exist real functions $h(x)$ and $\alpha(x)$ both of class $C^{\prime}$, as well as the quotient $\dot{h} / r$, such that

$$
\begin{gathered}
s[\alpha, x ; q]=h(x) \exp (i \alpha(x)), \\
\left(\frac{\dot{h}}{r}\right)^{\cdot}+\left(\left(1+b^{2}\right)-\left(\frac{\dot{\alpha}}{r}-b\right)^{2}\right) r h=0, \\
\frac{h^{2}(x) \dot{\alpha}(x)}{r(x)}=2 \int_{a}^{x} h \dot{h} b .
\end{gathered}
$$

Furthermore, if $b(x)$ is of class $C^{\prime}$ then

$$
h^{2}(x)\left(\frac{\dot{\alpha}(x)}{r(x)}-b(x)\right)=-\int_{a}^{x} h^{2}(t) \dot{b}(t) d t
$$

and $\dot{\alpha} / r$ is of class $C^{\prime}$, whenever $s \neq 0$.

Application of the Sturmian comparison theorem to $\left(12^{\prime}\right)$ gives the following.

CoRollary 1.1.1. (Non-oscillation theorem) If tho real second-order equation

$$
(\dot{y} / r)^{\cdot}+\left(1+b^{2}\right) r y=0, \quad a \leqq x<\infty .
$$

is non-oscillatory (i.e., non-trivial solution has infinitely many zeros on $a \leqq x<\infty)$ then $s[a, x ; q]$ is non-oscillatory.

Equation (14) shows the following.

CoRollary 1.1.2. (Non-oscillation theorem) If, in addition to the hypotheses of Theorem 1.1, $b(x)$ is of class $C^{\prime}$ and $b^{\prime}(x) \neq 0$ on $a<x<\infty$ then $s[a, x ; q]$ has no zeros of $a<x<\infty$.

EXXAPLE 1.1. Let $q(x)=1+i x$, then $r(x)=\sqrt{1+x^{2}}, \theta(x)=\operatorname{Tan}^{-1} x$, 
$b(x)=-\left(x^{2}+1\right)^{-3 / 2}$ and $\dot{b}(x)>0$. Corollary 1.1 .2 then establishes that $s[a, x ; q]$ has no zeros on $a<x<\infty$.

This example shows that the latter non-oscillation theorem is not a special case of the following.

ThEoREM T (Taam [8]). If $p(x)=p_{1}(x)+i p_{2}(x) \neq 0, f(x)=f_{1}(x)+i f_{2}(x)$, where $p_{1}, p_{2}, f_{1}$ and $f_{2}$ are real continuous functions on $a \leqq x \leqq b$ and there exist constants $j$ and $k$ and $a$ real function $m(x)$ of class $C^{\prime}$ on $a \leqq x \leqq b$ such that $j p_{1}(x)+k p_{2}(x)>0$

and

$$
\dot{m}+m^{2} /\left(j p_{1}+k p_{2}\right) \leqq-\left(j f_{1}+k f_{2}\right)
$$

on $a \leqq x \leqq b$

then the complex equation $(p(x) \dot{y})^{\circ}+f(x) y=0$ is disconjugate (i.e. no solution has two zeros on $a \leqq x \leqq b)$.

For $q(x)=1+i x$, as in Example 1.1, consider the equation $(\dot{y} / q)+\bar{q} y=$ 0 . Then

$$
p=\frac{1}{q}=\frac{\bar{q}}{|q|^{2}}=\frac{1-i x}{1+x^{2}}
$$

and $f(x)=\bar{q}=1-i x$. There exist constants $j, k$ with $k<0$ such that

$$
j p_{1}+k p_{2}=\frac{j+|k| x}{1+x^{2}}>0,
$$

for $x>j / k$. Consider the real second order equation

$$
\left(\frac{j+|k| x}{1+x^{2}} \dot{y}\right)^{\cdot}+(j+|k| x) y=0
$$

Now, $(j+|k| x)^{2} \leqq\left(k^{2}+j^{2}\right)\left(1+x^{2}\right)$ and, hence,

$$
\frac{j+|k| x}{1+x^{2}}<\frac{k^{2}+j^{2}}{j+|k| x}
$$

With the use of this inequality to increase the leading coefficient, equation (15) is altered to

$$
\left(\frac{k^{2}+j^{2}}{j+|k| x} \dot{y}\right)+(j+|k| x) y=0
$$

whose fundamental solutions are

$$
\sin \int_{a}^{x} \frac{j+|k| t}{\sqrt{j^{2}+k^{2}}} d t \text { and } \cos \int_{a}^{x} \frac{j+|k| t}{\sqrt{j^{2}+k^{2}}} d t
$$


and all solutions of $\left(15^{\prime}\right)$ oscillate on $j / k<a \leqq x<\infty$. By the Sturmian comparison theorem equation (15) is also oscillatory and hence for some $b>a$ there exists no function $m(x)$ required by the Riccati inequality of Theorem T. But by Corollary 1.1.2, $s[a, x ; q]$ has no zeros on $a<x<\infty$ and hence the complex equation $(\dot{y} / q)^{\circ}+\bar{q} y=0$ is disconjugate on $a \leqq x>\infty$. Therefore Example 1.1 is non-oscillatory but does not satisfy the hypotheses of Theorem $\mathrm{T}$.

REMARK. A similar polar form for the "cosine" functional

$$
c[a, x ; q]=k(x) \exp (i \beta(x))
$$

can be obtained for which $k$ and $\beta$ replace $h$ and $\alpha$, respectively, in equations $\left(12^{\prime}\right)$ and $\left(13^{\prime}\right)$. However, since $k(a)=1$, equation (14) must be replaced by

$$
k^{2}(x)\left(\frac{\dot{\beta}(x)}{r(x)}-b(x)\right)=-b(a)-\int_{a}^{x} k^{2}(t) \dot{b}(t) d t .
$$

Of course, $c[a, x ; q]$ can be calculated from a known $s[a, x ; q]$ by the derivative formula, $\dot{s}=q \bar{c}$, which is the process actually used in the succeeding discussion.

THEOREM 1.2. If, in addition to the hypotheses of Theorem 1.1, $b=\dot{\theta}(x) / 2 r(x)$ is constant then explicit solutions of the system (5) are

$$
\begin{gathered}
s[a, x ; q]=\frac{1}{\sqrt{b^{2}+1}} \exp \left(i \frac{\theta(x)+\theta(a)}{2}\right) \sin \phi(x), \\
c[a, x ; q]=\exp \left(i \frac{\theta(x)-\theta(a)}{2}\right)\left\{\cos \phi(x)-\frac{i b}{\sqrt{b^{2}+1}} \sin \phi(x)\right\},
\end{gathered}
$$

where $\phi(x)=\sqrt{b^{2}+1} \int_{a}^{x} r(t) d t$. Furthermore, $s[a, x, q]$ oscillates (i.e., has infinitely many zeros on $a \leqq x<\infty)$ if and only if $\int_{a}^{\infty}|r(t)| d t=\infty$. Final$l y$, if $b \neq 0, c[a, x ; q]$ has no zeros on $a \leqq x<\infty$.

Note that this means that there exists a second order complex equation $(\dot{y} / q)^{\cdot}+\bar{q} y=0$ such that the zeros of one solution do not separate those of a linearly independent solution and the zeros of a solution are not separated by zeros of its derivative. Before this theorem is proved consider the following special cases.

EXAMPLE 1.2. Let $q(x)$ be real and positive, then $q(x)=r(x), \theta(x)=0$, $b=0$ and 


$$
s[a, x ; q]=\sin \int_{a}^{x} q(t) d t, \quad c[a, x ; q]=\cos \int_{a}^{x} q(t) d t .
$$

ExAMPLE 1.3. Let $q(x)=q_{1}(x)+i q_{2}(x), q_{2}(x)=k q_{1}(x), k=$ constant, and $q_{1}(x)>0$ then $r(x)=\sqrt{ } 1+k^{2} \quad q_{1}(x), \theta(x)=\operatorname{Tan}^{-1} k, \dot{\theta}=0, b=0$ and

$$
\begin{aligned}
& s[a, x ; q]=\frac{1+i k}{\sqrt{1+k^{2}}} \cdot \sin \left(\sqrt{1+k^{2}} \int_{a}^{x} q_{1}(t) d t\right), \\
& c[a, x ; q]=\quad \cos \left(\sqrt{1+k^{2}} \int_{a}^{x} q_{1}(t) d t\right) .
\end{aligned}
$$

EXAMPLE 1.4. Let $q(x)=\exp (i x)$, then $r(x)=1, \theta(x)=x, b=1 / 2$ and

$$
\begin{gathered}
s[a, x ; q]=\frac{2}{\sqrt{5}} \exp \frac{i}{2}(x+a) \sin \frac{\sqrt{5}}{2}(x-a) \\
c[a, x ; q]=\exp \frac{i}{2}(x-a)\left(\cos \frac{\sqrt{5}}{2}(x-a)-\frac{i}{\sqrt{5}} \sin \frac{\sqrt{5}}{2}(x-a)\right) .
\end{gathered}
$$

Note that there do not exist constants $j, k$ such that $j q_{1}-k q_{2}>0$, and hence the uypotheses of Theorem $T$ are not satisfied for $b-a>\pi$. Of course, in this case $(\dot{y} / q)^{\circ}+\bar{q} y=0$ is oscillatory on $a \leqq x<\infty$.

Proof of THEOREM 1.2. In order to simplify computations let $\theta(a)=0$. There is no loss of substance decause of this assumption since Lemma 1.2 assures that if $q(x)$ is multiplied by the constant exp $(-i \theta(\alpha))$ then the resulting "sine" functional must be multiplied by that number and the "cosine" is unchanged. Therefore equation (14) gives

$$
\dot{\alpha}(x)=b r(x)=\frac{\dot{\theta}(x)}{2} \text { and } \alpha(x)=\frac{\theta(x)}{2},
$$

and equation $\left(12^{\prime}\right)$ becomes

$$
\left(\frac{\dot{h}}{r \sqrt{ } 1+b^{2}}\right)^{\cdot}+r \sqrt{1+b^{2}} h=0 .
$$

Since $h(a)=0$ and $\dot{h}(a)=r(a)$ we have

$$
h(x)=\frac{1}{\sqrt{ } b^{2}+1} \sin \left(\sqrt{1+b^{2}} \int_{a}^{x} r(t) d t\right) .
$$

By combination of (8), (18) and (20) and the use of Lemma 1.2 and $\dot{s}=$ $q \bar{c}$ the explicit solutions (16) and (17) are obtained. Finally, if $b \neq 0,(17)$ gives

$$
|c| \geqq 1-\frac{b^{2}}{b^{2}+1}=\frac{1}{b^{2}+1}>0
$$


and the theorem is proved.

Note that in Theorem 1.2 , if $b=0$ then $c[a, x ; q]$ oscillates if and only if $s[a, x ; q]$ oscillates and the zeros of one functional separate those of the other. But if $b \neq 0$ then $s[a, x ; q]$ may oscillate but $c[a, x ; q]$ has no zeros on $a \leqq x<\infty$, thus violating a "Rolle's Theorem" for complex functions.

In the next section it will be shown that every complex equation of the form $(p \dot{y})^{*}+f y=0$ can be transformed into the "special" form $(\dot{y} / q)^{\cdot}+\bar{q} y=0$.

2. A complex Prüfer transformation. Consider the complex general linear second-order equation

$$
(p \dot{y})^{\cdot}+f y=0,
$$$$
a \leqq x<\infty,
$$

where $p=p_{1}(x)+i p_{2}(x) \neq 0, f=f_{1}(x)+i f_{2}(x)$ and $p_{1}, p_{2}, f_{1}, f_{2}$ are all real continuous functions on $a \leqq x<\infty$. Suppose $y(x)$ is a non-trivial solution of (1) such that $y(\alpha)=0$ and there exist complex functions $\rho(x) \neq 0, w(x) \neq 0$ of class $C^{\prime}$ and $q(x)$ continuous, such that

$$
\begin{aligned}
y(x) & =\rho(x) s[a, x ; q], \\
p(x) \dot{y}(x) & =\bar{w}(x) \bar{\rho}(x) \bar{c}[a, x ; q] .
\end{aligned}
$$

Then by differentiating both equations of (21) and combining with (1) we obtain

$$
\begin{gathered}
\dot{\rho} s+\rho q \bar{c}=\frac{\bar{w}}{p} \overline{\rho c}, \\
\dot{\rho} c-\rho q \bar{s}=-\frac{\bar{f}}{w} \bar{\rho} \bar{s}-\frac{\dot{w}}{w} \rho c .
\end{gathered}
$$

Hence, solving for $\dot{\mu}$ and $q$ and recalling that $|c|^{2}+|s|^{2}=1$ we have

$$
\begin{aligned}
& \dot{\rho}=\overline{\rho s c}\left(\frac{\bar{w}}{p}-\frac{\bar{f}}{w}\right)-\frac{\dot{w}}{w}|c|^{2} \rho, \quad \bar{\rho}(a)=\frac{p(a) \dot{y}(a)}{\bar{w}(a)} \neq 0 \\
& q=\frac{\bar{\rho}}{\rho}\left(\frac{\bar{w}}{p}|c|^{2}+\frac{\bar{f}}{w}|s|^{2}\right)+\frac{\dot{w}}{w} s c
\end{aligned}
$$

For $p, f, y, \rho, q$ real ; $s=\sin \int_{a}^{x} q, c=\cos \int_{a}^{x} q$ and (21), (22), (23) reduce to the modified real Prüfer transformation of [1]. The transformation of (1) given by $y=\rho s$ and $\dot{p} y=\rho c$ results in the differential-integral system $\dot{\rho}=\rho(1 / p) c \bar{s}-f s \bar{c})$ and $q=(1 / p) c^{2}+f s^{2}$ and can be obtained as a direct application of the matrix Prüfer transformation in [3] for (matrix) order 2. However, the form (21) seems to be more useful, e.g., see Corol- 
lary 2.1.

Consider next the question of existence of $\rho(x)$ and $q(x)$, that is the solution pair of the system (22), (23). The method is that of successive approximations and the following lemmas establish a Lipschitz condition for the system.

Lemma 2.1. If $q(x)$ and $q^{*}(x)$ are continuous complex functions on $a \leqq x<\infty$ and $s^{*}=s\left[a, x ; q^{*}\right], c^{*}=c\left[a, x ; q^{*}\right]$ then

$$
\left.\left|s-s^{*}\right|\right\}\left|\leqq 4 \int_{a}^{x}\right| q-q^{*} \mid, \quad a \leqq x<\infty .
$$

Proof. By subtracting the differential equations (5) obtain the system

$$
\begin{aligned}
& \left(s-s^{*}\right)^{\cdot}=q\left(\overline{c-c^{*}}\right)+\left(q-q^{*}\right) \bar{c}^{*}, \\
& \left(c-c^{*}\right)^{\cdot}=-q\left(\overline{s-s^{*}}\right)-\left(q-q^{*}\right) \bar{s}^{*},
\end{aligned}
$$

which can be expressed in the vector-matrix form

$$
\begin{aligned}
& \dot{\alpha}=Q(x) \alpha+\beta(x), \alpha=\left(\frac{s-s^{*}}{c-c^{*}}\right), Q=\left(\begin{array}{cr}
0 & q(x) \\
-\bar{q}(x) & 0
\end{array}\right), \beta=\left(\begin{array}{c}
\left(q-q^{*}\right) \bar{c}^{*} \\
\left(\overline{\left.q-q^{*}\right)} s^{*}\right.
\end{array}\right) \\
& \alpha(a)=0 .
\end{aligned}
$$

Let $Y(x)$ be the matrix solution of the homogeneous equation:

$$
\dot{Y}=Q Y, Y(a)=E=\left(\begin{array}{ll}
1 & 0 \\
0 & 1
\end{array}\right) \text {. }
$$

Then

$$
Y(x)=\left(\begin{array}{ll}
c & s \\
-\bar{s} & \bar{c}
\end{array}\right) \text {, and } Y^{-1}=\left(\begin{array}{rr}
\bar{c} & -s \\
\bar{s} & c
\end{array}\right)
$$

By elementary methods the solution of (21) is

$$
\alpha(x)=\int_{a}^{x} Y(x) Y^{-1}(t) \beta(t) d t .
$$

Hence, by taking norms (square root of sum of square of absolute values), we have

$$
\|\alpha(x)\| \leqq 2 \int_{a}^{x}\|\beta(t)\| d t<4 \int_{a}^{x}\left|q-q^{*}\right|,
$$

from which the conclusion of the lemma follows.

Lemma 2.2. Assume the hypotheses of Lemma 2.1 and let 


$$
r[q]=\sum_{i=1}^{n} k_{i}(x) s^{\alpha} \cdot(\bar{s})^{\beta}{ }_{i} c^{\gamma} i(\bar{c})^{\delta_{i}},
$$

where $k_{i}(x)(i=1,2, \cdots, n)$ are complex continuous function on $a \leqq x \leqq b$ and $a_{i}, \beta_{i}, \gamma_{i}, \delta_{i}$ are non-negative integers. Then there exists a positive constant $K_{0}$ (independent of $q$ and $q^{*}$ ) such that

$$
\left|r[q]-r\left[q^{*}\right]\right| \leqq K_{0} \int_{a}^{x}\left|q-q^{*}\right|, \quad a \leqq x \leqq b .
$$

The proof based on Lemma 2.1 is simple and is omitted.

Lemma 2.3. If $u(x)$ and $v(x)$ are complex continuous functions on $a \leqq x<\infty$, the complex differential equation (see equation (22))

$$
\dot{\rho}=u(x) \bar{\rho}+v(x) \rho
$$

has exactly one solution for a prescribed value of $\rho(a)$.

The proof parallels that for real linear equations and, consequently, is not given here.

Lemma 2.4. Let $\rho(x)$ be a solution of (27), where $u$ and $v$ are the corresponding coefficients of (22), and $m(x)=\bar{\rho} \mid \rho$. Then: (i ) $|m| \equiv 1$, (ii) $m$ satisfies the complex Riccati equation

$$
\dot{m}=\bar{u}-(v-\bar{v}) m-u m^{2}
$$

and (iii) if $m^{*}$ is the corresponding function when $q$ is replaced by $q^{*}$, $u$ by $u^{*}$ and $v$ by $v^{*}$ then there exists a real constant $K_{1}$ (independent of $q$ and $\left.q^{*}\right)$ such that

$$
\left|m-m^{*}\right| \leqq K_{1} \int_{a}^{x}\left|q(t)-q^{*}(t)\right| d t, \quad a \leqq x<x_{1}<\infty .
$$

$$
\begin{aligned}
& \left(m-m^{*}\right) \cdot+\left\{(v-\bar{v})+u\left(m+m^{*}\right)\right\}\left(m-m^{*}\right) \\
= & \left(\overline{u-u^{*}}\right)+\left\{(\bar{v}-v)-\left(\bar{v}^{*}-v^{*}\right)\right\} m^{*}-\left(u-u^{*}\right) m^{* 2}
\end{aligned}
$$

or

$$
\left(m-m^{*}\right)^{\cdot}+n(x)\left(m-m^{*}\right)=r(x), \quad m(a)-m^{*}(a)=0,
$$

and hence,

$$
m(x)-m^{*}(x)=e^{-\int_{a}^{x} \int_{a}^{x} e \int_{a^{n}}^{t_{n}} r(t) d t .}
$$

Therefore, there exists a real constant $K_{1}$ such that

$$
\left|m(x)-m^{*}(x)\right| \leqq K_{1} \int_{a}^{x}\left|q-q^{*}\right|, \quad a \leqq x \leqq x_{1}<\infty .
$$


LEMMA 2.5. There exists a unique solution pair $\rho(x), q(x)$ of the system $(22,23)$.

Proof. It follows easily from Lemmas 2.2 and 2.4 that the system ((22), (23)) satisfies a Lipschitz condition. Let $q_{0}(x)$ and $\rho_{0}(x)$ be complex continuous functions on $a \leqq x \leqq b$ and for each non-negative integer $n$

$$
\begin{aligned}
& \rho_{n+1}(x)=\rho(a)+\int_{a}^{x}\left\{\bar{\rho}_{n} \bar{s}_{n} \bar{c}_{n}\left(\frac{\bar{w}}{p}-\frac{\bar{f}}{w}\right)-\frac{\dot{w}}{w}\left|c_{n}\right|^{2} \rho_{n}\right\}, \\
& q_{n+1}(x)=\frac{\bar{\rho}_{n}}{\rho_{n}}\left\{\frac{\bar{w}}{p}\left|c_{n}\right|^{2}+\frac{\bar{f}}{w}\left|s_{n}\right|^{2}\right\}+\frac{\dot{w}_{s} c_{n},}{w},
\end{aligned}
$$

where $s_{n}=s\left[a, x ; q_{n}\right]$ and $c_{n}=\left[a, x ; q_{n}\right]$.

By the usual successive approximation arguments it follows that the sequences $\left\{\rho_{n}(x)\right\}$ and $\left\{q_{n}(x)\right\}$ converge uniformly on $a \leqq x \leqq b$ to continuous limit functions, $\rho(x)$ and $q(x)$, respectively, which form a solution pair of (22) and (23).

THEOREM 2.1. If $y(x)$ is a non-trivial solution of (1), such that $y(a)=0$, and $w(x)$ is an arbitrary non-zero function of class $C^{\prime}$ then there exist a non-zero function $\rho(x)$ of Class $C^{\prime}$ and a continuous function $q(x)$ such that (21) is satisfied. Furthermore, (22) and (23) are satisfied.

Proof. From Lemma 2.5, there exists a unique solution pair $\rho(x)$ and $q(x)$. Let $u(x)=\rho(x) s[a, s ; q]$, then $u(a)=0=y(a)$,

$$
\dot{u}=\dot{\rho} s+\rho q \bar{c}=\frac{\bar{\rho} \bar{w}}{p} \bar{c} \text {, and } \dot{u}(a)=\frac{\bar{\rho}(a) \bar{w}(a)}{p(a)}=\dot{y}(a) \text {. }
$$

Finally,

$$
(p \dot{u})^{\cdot}=-\bar{\rho} \bar{w} \bar{q} s+\dot{\bar{w}} \bar{\rho} \bar{c}+\dot{\bar{\rho}} \bar{w} \bar{c}=-f \rho s=-f u,
$$

therefore

$$
y(x) \equiv u(x)=\rho(x) s[a, x ; q] .
$$

Equation (22) yields the following bounds on solutions

Corollary 2.1. (i ) $|\rho|=\sqrt{|y|^{2}+\left|\frac{p \dot{y}}{w}\right|^{2}}$.

$$
|y(x)| \leqq|\rho(x)| \leqq|\rho(a)| \exp \int_{a}^{x}\left\{\frac{1}{2}\left|\frac{\bar{w}}{p}-\frac{\bar{f}}{w}\right|+\mid \frac{\dot{w}}{w}\right\}
$$


(iii) if $w=k$, a real positive constant, then

$$
\begin{aligned}
|y(x)| \leqq & \rho(\alpha)\left|\exp \frac{1}{2} \int_{a}^{x}\right| \frac{k}{p}-\frac{\bar{f}}{k} \mid \leqq \\
& |\rho(\alpha)| \exp \frac{1}{2} \int_{a}^{x}\left\{\left|\frac{k p_{1}}{|p|^{2}}-\frac{f_{1}}{k}\right|+\mid \frac{k p_{2}}{|p|^{2}}-\frac{f_{2}}{k}\right\}
\end{aligned}
$$

Proof. (i) is obvious and (ii) follows directly from (22). (iii) results from an application of (ii) and a simple inequality about complex numbers. Note that if $p$ is real then $p_{2}=0$ and (31) becomes

$$
|y(x)| \leqq|\rho(a)| \exp \frac{1}{2} \int_{a}^{x}\left\{\left|\frac{k}{p}-\frac{f_{1}}{k}\right|+\left|\frac{f_{2}}{k}\right|\right\},
$$

which is the " non-symmetric" bound given by Taam [10].

Finally, other choices of $w(x)$ give other bounds on solutions as was found for real second-order differential equations in [1].

\section{REFERENCES}

1 J. H. Barrett, Behavior of solutions of second order self-adjoint differential equations, Proc. Amer. Math. Soc. 6 (1955), 247-251.

2. - Matrix systems of second order differential equations, Portugaliae Math. 14 (1955), 79-89.

$3 . \quad-$ A Prüfer transformation for matrix differential equations Proc. Amer. Math. Soc. 8 (1957), 510-518.

4. E. Hille, Oscillation theorems in the complex domain, Trans. Amer. Math. Soc. 23 (1922), 350-385.

5. Behavior of solutions of linear second order differential equations, Ark. Mat, 2, (1951), 25-41.

6. H. Prüfer, Neue Herleitung der Sturm-Liouvilleschen Reihenentwicklung stetiger Funktionen, Math. Ann. 95 (1926), 499-518.

7. C. T. Taam, The boundedness of the solutions of a differential equation in the complex domain, Pacific J. Math. 2 (1952), 643-654.

8. - On the solutions of second order linear differe tial equations, Proc. Amer. Math. Soc. 4 (1953), 876-879.

9 . , Oscillation theorems. Amer. J. Math. 74 (1952), 387-424.

10. On the complcx zeros of functions of Sturm-Liouville type, Pacific J. Math. 3 (1953), 837-843.

UNIVERSITY OF UTAH 


\section{PACIFIC JOURNAL OF MATHEMATICS}

\section{EDITORS}

\section{H. L. Royden}

Stanford University

Stanford, California

\section{R. A. Beaumont}

University of Washington

Seattle 5 , Washington

\author{
A. L. Whiteman
}

University of Southern California

Los Angeles 7, California

E. G. Straus

University of California

Los Angeles 24, California

\section{ASSOCIATE EDITORS}
E. F. BECKENBACH
A. HORN
L. NACHBIN
G. SZEKERES
C. E. BURGESS
V. GANAPATHY IYER
I. NIVEN
F. WOLF
M. HALL
R. D. JAMES
T. G. OSTROM
E. HEWITT
M. S. KNEBELMAN
M. M. SCHIFFER
K. YOSIDA

\section{SUPPORTING INSTITUTIONS}

\author{
UNIVERSITY OF BRITISH COLUMBIA \\ CALIFORNIA INSTITUTE OF TECHNOLOGY \\ UNIVERSITY OF CALIFORNIA \\ MONTANA STATE UNIVERSITY \\ UNIVERSITY OF NEVADA \\ OREGON STATE COLLEGE \\ UNIVERSITY OF OREGON \\ UNIVERSITY OF SOUTHERN CALIFORNIA
}

\author{
STANFORD UNIVERSITY \\ UNIVERSITY OF UTAH \\ WASHINGTON STATE COLLEGE \\ UNIVERSITY OF WASHINGTON \\ * * * * \\ AMERICAN MATHEMATICAL SOCIETY \\ CALIFORNIA RESEARCH CORPORATION \\ HUGHES AIRCRAFT COMPANY \\ THE RAMO-WOOLDRIDGE CORPORATION
}

Mathematical papers intended for publication in the Pacific Journal of Mathematics should be typewritten (double spaced), and the author should keep a complete copy. Manuscripts may be sent to any of the editors. All other communications to the editors should be addressed to the managing editor, E. G. Straus at the University of California, Los Angeles 24, California.

50 reprints per author of each article are furnished free of charge; additional copies may be obtained at cost in multiples of 50 .

The Pacific Journal of Mathematics is published quarterly, in March, June, September, and December. The price per volume (4 numbers) is $\$ 12.00$; single issues, $\$ 3.50$. Back numbers are available. Special price to individual faculty members of supporting institutions and to individual members of the American Mathematical Society: $\$ 4.00$ per volume; single issues, $\$ 1.25$.

Subscriptions, orders for back numbers, and changes of address should be sent to Pacific Journal of Mathematics, 2120 Oxford Street, Berkeley 4, California.

Printed at Kokusai Bunken Insatsusha (International Academic Printing Co., I.td.), No. 10, 1-chome, Fujimi-cho, Chiyoda-ku, Tokyo, Japan.

PUBLISHED BY PACIFIC JOURNAL OF MATHEMATICS, A NON-PROFIT CORPORATION

The Supporting Institutions listed above contribute to the cost of publication of this Journal, but they are not owners or publishers and have no responsibility for its content or policies. 


\section{Pacific Journal of Mathematics}

\section{Vol. 8, No. 2 \\ April, 1958}

John Herbert Barrett, Second order complex differential equations with a real independent variable ............................ 187

Avner Friedman, Remarks on the maximum principle for parabolic equations and its applications ......................... 201

Richard Robinson Goldberg, An inversion of the Stieltjes transform ....... 213

Olavi Hellman, On the periodicity of the solution of a certain nonlinear integral equation .................................. 219

Gilbert Helmberg, A theorem on equidistribution on compact groups...... 227

Lloyd Kenneth Jackson, Subfunctions and the Dirichlet problem ......... 243

Naoki Kimura, The structure of idempotent semigroups. I ............ 257

Stephen Kulik, A method of approximating the complex roots of equations........................................ 277

Ancel Clyde Mewborn, A note on a paper of L. Guttman.............. 283

Zeev Nehari, On the principal frequency of a membrane ............ 285

G. Pólya and I. J. Schoenberg, Remarks on de la Vallée Poussin means and convex conformal maps of the circle ...................... 295

B. M. Stewart, Asymmetry of a plane convex set with respect to its centroid .......................................... 335

Hans F. Weinberger, Lower bounds for higher eigenvalues by finite difference methods

Edwin Weiss and Neal Zierler, Locally compact division rings ......... 369

Bertram Yood, Homomorphisms on normed algebras ................. 373 